\title{
Note
}

\section{Cycloalliin Inhibits Melanin Biosynthesis in B16 Mouse Melanoma Cells}

\author{
Tomohiro Bito", Kyohei Koseki, Tomohiro Moriguchi, Yu Sasaki, Yukinori Yabuta, Tsuyoshi IchiYanagi, \\ and Fumio Watanabe
}

Department of Agricultural, Life and Environmental Sciences, Faculty of Agriculture, Tottori University, Tottori 680-
8553, Japan

Received February 9, 2018 ; Accepted March 28, 2018

We evaluated cycloalliin, a sulfur-containing imino acid found in garlic and onion, for its possible use as an ingredient in cosmetics. The activity of tyrosinase, an enzyme involved in melanin synthesis, was inhibited by cycloalliin in a dose-dependent manner. Cycloalliin was also found to be a mixed-type inhibitor of the monophenolase and diphenolase activities of tyrosinase with $K_{\mathrm{i}}$ values of $56.0 \mathrm{mM}$ and $13.6 \mathrm{mM}$, respectively. In addition, cycloalliin only weakly inhibited the activity of mushroom tyrosinase. Furthermore, cycloalliin significantly reduced $\alpha$-melanocyte-stimulating hormone-induced melanin levels and both protein and mRNA levels of tyrosinase in B16 mouse melanoma cells at a final concentration of $3.8 \mu \mathrm{M}$. Cycloalliin also significantly inhibited the activity of adenylate cyclase, an enzyme involved in the cyclic adenosine monophosphate-signaling pathway during melanogenesis, and consequently mRNA and protein expression of tyrosinase was reduced significantly. These results show that cycloalliin may be useful as a skin-lightening agent in the cosmetics industry.

Keywords: B16 mouse melanoma cells, cyclic AMP signaling pathway, cycloalliin, onion, tyrosinase

\section{Introduction}

Onion (Allium cepa L.) is a globally important vegetable and is cultivated over a wide area (Benítez et al., 2011), and its relatives include garlic (Allium sativum L.). They both contain various bioactive compounds, particularly organosulfur compounds, which are beneficial to human health (VazquezPrieto and Miatello, 2010). Of these, cycloalliin is a sulfurcontaining imino acid (Figure 1) that is present in low levels in fresh onions, but increases significantly during heat processing (Ueda et al., 1994). This stable and flavorless compound can reduce serum triacylglycerols in rats (Yanagita et al., 2003) and induce NADPH-quinone reductase involved in protection against toxic and carcinogenic chemicals (Xiao and Parkin, 2002). Moreover, cycloalliin significantly increases fibrinolytic activity in human blood (Agarwal et al., 1977). Recently, efficient methodologies to optimally extract cycloalliin from garlic bulbs were reported (Lee et al., 2013, Lee et al., 2016). Our preliminary study on the beneficial properties of cycloalliin indicated that cycloallin significantly inhibits the activity of

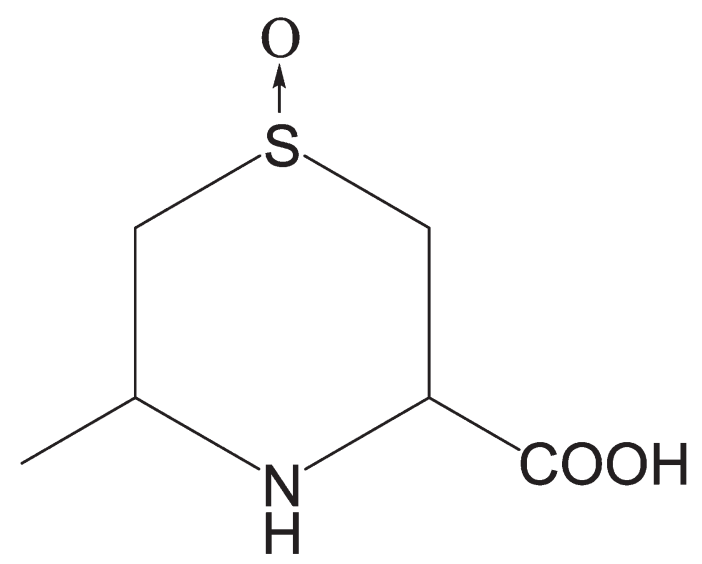

Fig. 1. Diagrammatic representation of the structure of cycloalliin.

tyrosinase, which is involved in melanogenesis.

Although melanin is crucial in protecting the skin against UV-induced injury, its aberrant functioning and accumulation in the skin causes hyperpigmentation disorders, such as 
melasma, freckles, and geriatric pigment spots (Kim et al., 2005). Melanogenesis is regulated by tyrosinase, tyrosinaserelated protein 1 (TRP1), and tyrosinase-related protein 2 (TRP2) (Kameyama et al., 1995). In particular, tyrosinase is a key enzyme that catalyzes the first two steps in melanin synthesis: the hydroxylation of tyrosine to 3-(3,4-dihydroxyphenyl)- L-alanine (L-DOPA) and the oxidation of L-DOPA to dopaquinone. Several tyrosinase inhibitors have been used as skin-lightening agents in the cosmetics industry (Loizzo et al., 2012). Melanin production is mainly regulated in skin melanocytes by $\alpha$-melaonocytestimulating hormone $(\alpha-\mathrm{MSH})$, which binds to the melanocortin receptor 1 (MC1R) on the membrane of melanocytes and then activates adenylate cyclase (AC) to form cyclic adenosine monophosphate (cAMP), which in turn acts as a second intracellular messenger. cAMP stimulates the phosphorylation of protein kinase A (PKA), which induces the expression of microphthalmia-associated transcription factor (MITF), a master transcription factor in melanogenesis (Chang, 2012), through the phosphorylation of cAMP response element-binding protein (CREB). The upregulation of MITF stimulates the expression of tyrosinase, TRP1, and TRP2. To date, various compounds with the capability to decrease the expression of MITF have been isolated from natural resources and characterized.

As per our knowledge, to date, no study has investigated the potential of cycloalliin to be used in cosmetics. Therefore, in the present study, we examined the inhibitory effects of cycloalliin on the catalytic activity of tyrosinase and cAMPdependent pathway involved in melanogenesis using in B16 mouse melanoma cells.

\section{Materials and Methods}

Materials Dulbecco's Modified Eagle's Medium (DMEM), mushroom tyrosinase, and synthetic melanin were purchased from Sigma-Aldrich Japan, Inc. (Tokyo, Japan). $\alpha$-MSH and L-DOPA were purchased from Wako Pure Chemical Industries, Ltd. (Osaka, Japan). Phosphate-buffered saline (PBS) and trypsin solution used for cell culture were obtained from Life Technologies Corp. (Carlsbad, CA). Cycloalliin was synthesized using a previously published method (Sakai and Yoneda, 1981) and was validated by comparing its ${ }^{1} \mathrm{H}-\mathrm{NMR}$ spectrum with that of authentic cycloalliin (Wako) using ${ }^{1} \mathrm{H}-\mathrm{NMR}$ spectroscopy. All other reagents were of the highest commercially available purity.

Cell culture B16 melanoma (murine melanin-producing melanoma, RCB1283) cells were purchased from Riken Bio Resource Center (Tsukuba, Japan). B16 cells were cultured in DMEM supplemented with $10 \%(\mathrm{v} / \mathrm{v})$ fetal bovine serum (Biowest, Paris, France) and $1 \%(\mathrm{v} / \mathrm{v})$ penicillin/streptomycin (Wako) in an incubator with $5 \% \mathrm{CO}_{2}$ at $37{ }^{\circ} \mathrm{C}$. During incubation, the medium was changed every 3 days.

Assessing inhibitory activity of tyrosinase Cycloalliin was dissolved in dimethyl sulfoxide (DMSO) to be used for the experiments. To determine the effects of cycloalliin on the diphenolase activity of tyrosinase, the reaction mixture we used contained $667 \mu \mathrm{L}$ of $0.1 \mathrm{M} 4$-(2-hydroxyethyl)-1-piperazine ethane sulfonic acid (HEPES) buffer ( $\mathrm{pH} 7.0$ ), $200 \mu \mathrm{L}$ of $2.5-$ $20 \mathrm{mM}$ L-DOPA dissolved in HEPES buffer (at a final concentration of $0.5-4 \mathrm{mM}$ ), and $100 \mu \mathrm{L}$ of $54 \mathrm{mM}$ or $108 \mathrm{mM}$ cycloalliin solution (at a final concentration of $5.4 \mathrm{mM}$ or $10.8 \mathrm{mM}$, respectively). The cycloalliin solutions contained $30 \%(\mathrm{v} / \mathrm{v})$ DMSO, which was also used on its own in the control mixture instead of the cycloalliin solution. Mixtures were preincubated for $1 \mathrm{~min}$ at $30{ }^{\circ} \mathrm{C}$; subsequently, the enzymatic reaction was initiated by the addition of $33 \mu \mathrm{L}$ of mushroom tyrosinase ( $1.7 \mathrm{units} / \mathrm{mL}$ of reaction mixture), which was dissolved in the identical HEPES buffer. The mixtures were allowed to stand for $2 \mathrm{~min}$. Changes in absorbance during the experimental period were measured at $475 \mathrm{~nm}$ using a Shimadzu spectrophotometer (UV-2550). For assessing the effects of cycloallin on the monophenolase activity of tyrosinase, the reaction mixture we used contained $40 \mu \mathrm{L}$ of identical HEPES buffer, $20 \mu \mathrm{L}$ of $0.5-10 \mathrm{mM}$ L-tyrosine dissolved in the identical HEPES buffer (at a final concentration of $0.1-2 \mathrm{mM}$ ), and $20 \mu \mathrm{L}$ of $108 \mathrm{mM}$ or $216 \mathrm{mM}$ cycloalliin solution (at a final concentration of $21.6 \mathrm{mM}$ or $43.2 \mathrm{mM}$, respectively). This cycloalliin solution contained $60 \%(\mathrm{v} / \mathrm{v})$ DMSO, which was used on its own as a control instead of cycloalliin solution in the main mixture. The enzymatic reaction was initiated by addition of $20 \mu \mathrm{L}$ of mushroom tyrosinase ( 5 units $/ \mathrm{mL}$ of reaction mixture), which was dissolved in HEPES buffer and allowed to stand for $2 \mathrm{~min}$. Changes in absorbance during the experimental period were measured at $475 \mathrm{~nm}$ using a Sunrise Rainbow RC-R microplate reader (Tecan Austria GmbH, Salzburg, Austria).

The inhibitory activity of tyrosinase was estimated considering the activity of tyrosinase for L-DOPA or L-tyrosine in the presence and absence of cycloalliin. The $K i$ values of cycloalliin were calculated using the Lineweaver-Burk plot (Lineweaver and Burk, 1934).

Assessing inhibitory activity of cycloalliin against melanin using cell culture system Cycloalliin was dissolved in DMSO and diluted with distilled water to final concentrations of $0.1 \%$, $1 \%, 5 \%$, and $10 \%(\mathrm{v} / \mathrm{v})$. During our preliminary experiments, we found that a DMSO concentration of less than $3.4 \times 10^{-4}$ percent did not affect cell viability, which was analyzed using trypan blue dye exclusion staining with a cell counter (TC20 ${ }^{\mathrm{TM}}$, Bio-Rad Laboratories Inc., Hercules, CA). Briefly, melanin levels were determined as follows: B16 cells were cultured in the presence of $10 \mathrm{nM} \alpha$-MSH with $5.0 \times 10^{4}$ cells/well in 12 well plates. After $24 \mathrm{~h}$, cells in each well were treated with $20 \mu \mathrm{L}$ of cycloalliin solution and further grown for $72 \mathrm{~h}$. After washing with PBS, cells were harvested by trypsinization. Each cell pellet was treated with $1 \mathrm{~mL}$ of $1 \mathrm{M} \mathrm{NaOH}$ at $80{ }^{\circ} \mathrm{C}$ for $1 \mathrm{~h}$. The absorbance of each cell extract was measured at $475 \mathrm{~nm}$ 
Table 1. Primer pairs used for quantitative polymerase chain reaction (qPCR) analysis

\begin{tabular}{|c|c|}
\hline Name & Primer sequences $\left(5^{\prime}-3^{\prime}\right)$ \\
\hline \multirow[t]{2}{*}{ Tyrosinase } & Forward: CAGATCTCTGATGGCCAT \\
\hline & Reverse: GGATGACATAGACTGAGC \\
\hline \multirow[t]{2}{*}{ TRP1 } & Forward: CTTTCTCCCTTCCTTACTGG \\
\hline & Reverse: TGGCTTCATTCTTGGTGCTT \\
\hline \multirow[t]{2}{*}{ TRP2 } & Forward: TGAGAAGAAACAAAGTAGGCAGAA \\
\hline & Reverse: CAACCCCAAGAGCAAGACGAAAGC \\
\hline \multirow[t]{2}{*}{ MITF } & Forward: TTATAGTACCTTCTCTTTGCCAGTCC \\
\hline & Reverse: GTTTATTTGCTAAAGTGGTAGAAAGGTACT \\
\hline \multirow[t]{2}{*}{ GAPDH } & Forward: GGAGAAGGCTGGGGCTCAT \\
\hline & Reverse: TGATGGCATGGACTGTGGTC \\
\hline
\end{tabular}

The primer pairs for qPCR were designed using GENETYX. For normalization, GAPDH served as the internal standard.

using a Shimadzu spectrophotometer (UV-2550). Synthetic melanin was used as a standard.

Quantitative PCR analysis ( $q P C R$ ) Total RNA was prepared from the cell solution using Sephasol ${ }^{\circledR}$-RNA1 (Nacalai Tesque Inc.). Total RNA was used to synthesize cDNA using PrimeScript ${ }^{\mathrm{TM}}$ RT reagent kit with gDNA Eraser (Takara Bio, Otsu, Japan). Primer pairs used for qPCR analysis were designed using GENETYX software (GENETYX Corporation, Tokyo, Japan) to yield 18-30-nucleotide sequences with amplification products approximately $100 \mathrm{bp}$ long (Table 1). A CFX Connect ${ }^{\mathrm{TM}}$ Real-Time System (BioRad) with SYBR Premix Ex Taq (Takara Bio) was used to perform qPCR. mRNA levels required for encoding glyceraldehyde-3-phosphate dehydrogenase (GAPDH) were used as an internal standard. The qPCR experiments were repeated at least three times for each cDNA prepared from three preparations of cells.

Western blot analysis After reaching confluence, cells were harvested and dissolved with passive lysis buffer (Promega Corp, Madison, WI) on ice for $30 \mathrm{~min}$. The solution was centrifuged at $10,000 \times g$ for $10 \mathrm{~min}$ at $4{ }^{\circ} \mathrm{C}$, and the supernatant fraction was used as a crude enzyme solution for western blotting. The cell solution was separated with sodium dodecyl sulfate (SDS-) polyacrylamide gel electrophoresis. We used a precast slab gel (e-PAGEL, model E-R520L; ATTO Corporation, Tokyo, Japan) for electrophoresis of samples through a $5 \%-20 \%(\mathrm{w} / \mathrm{w})$ linear gradient of polyacrylamide in the presence of SDS. After electrophoresis, the proteins were transferred onto a polyvinylidene difluoride (PVDF) membrane (Immuno-Blot PVDF; Bio-Rad) in a Trans-Blot SD semi-dry electrophoretic transfer cell (Bio-Rad). Non-specific binding was blocked using $5 \%(\mathrm{w} / \mathrm{v})$ non-fat dry milk in PBS/Tween 20 buffer. PVDF membranes were probed with anti-CREB phosphorylated antibody (serine 133) (ab32096, Abcam), antiCREB (total CREB) antibody (ab178322, Abcam), and antiGAPDH antibody (ab8245, Abcam). We performed the immunodetection reactions using an anti-mouse IgG secondary antibody coupled to horseradish peroxidase (ab6721, Abcam) and tetramethylbenzidine, a stabilized substrate for horseradish peroxidase (Promega, Tokyo, Japan), according to the manufacturer's instructions. A protein ladder (Amersham ${ }^{\mathrm{TM}}$ ECL $^{\text {TM }}$ Rainbow ${ }^{\mathrm{TM}}$ Marker-Full Range; GE Healthcare, Minneapolis, MN) was used to determine molecular mass.

Other assays The intracellular levels of cAMP were quantified using a cAMP enzyme immunometric assay kit (R\&D systems, Inc., Minneapolis, MN), according to the manufacturer's instructions. Adenylate kinase activity was assessed using adenosine triphosphate (ATP), which was generated from adenosine diphosphate (ADP) as a substrate using an AC activity kit (BioVision, Milpitas, CA), according to the manufacturer's instructions. PKA activity was assayed using a PKA kinase activity kit (Enzo Life Sciences, NY).

Statistical analyses B16 cell viability, melanin concentration, cAMP levels, AC and PKA activity, and mRNA levels of proteins playing a role in melanogenesis via the cAMP-dependent signaling pathway were analyzed using oneway ANOVA with Tukey's multiple comparison test. Analyses were performed using GraphPad Prism ${ }^{\circledR}$ for Windows, version 5.03 (GraphPad Software Inc., La Jolla, CA). All data were expressed as means \pm standard deviation, and statistically significant differences were defined at $p<0.05$.

\section{Results and Discussion}

Cycloalliin weakly inhibited the activity of mushroom tyrosinase activity Tyrosinase catalyzes the hydroxylation of tyrosinase to form L-DOPA (monophenolase activity) and then the oxidation of L-DOPA to form dopaquinone (diphenolase activity), which changes into melanin after several spontaneous chemical reactions. Therefore, the effect of varied concentrations of cycloalliin on the oxidation of L-DOPA (diphenolase activity) was studied using mushroom tyrosinase, which is generally used in the experiment of inhibition of 


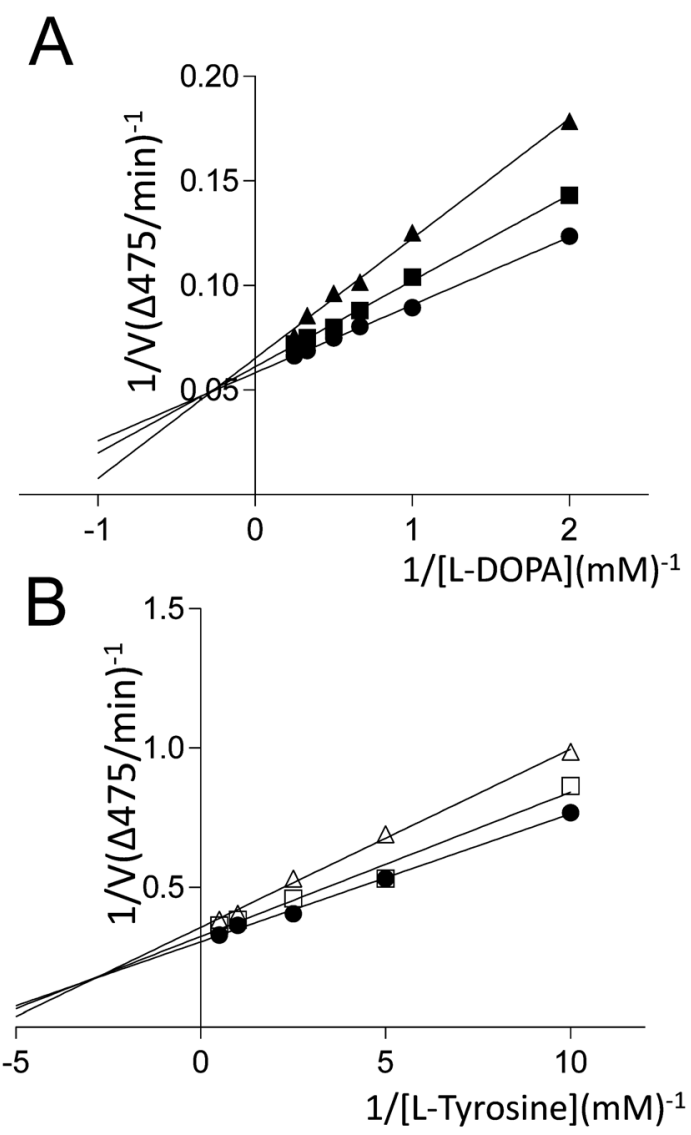

Fig. 2. Lineweaver-Burk double reciprocal plot of the activity of mushroom tyrosinase in the presence of cycloalliin. Absorbancechange rate $(\mathrm{V})$ was measured with various concentrations of L-DOPA and L-tyrosine as substrates for diphenolase activity (A) and monophenolase activity (B), respectively. Cycloalliin was used at concentrations of $0 \mathrm{mM}(\mathbf{O}), 5.4 \mathrm{mM}(\boldsymbol{\square}), 10.8 \mathrm{mM}(\boldsymbol{\Delta})$, $108 \mathrm{mM}(\square)$, and $216 \mathrm{mM}(\triangle)$. Data were obtained for three independent experiments.

melanin biosynthesis. The diphenolase activity of tyrosinase was inhibited in a dose-dependently the addition of cycloalliin, which showed a mixed inhibitory effect on the enzyme's overall activity. The $K$ i value of cycloalliin was calculated to be $13.6 \mathrm{mM}$ (Figure 2A). The monophenolase activity of tyrosinase was determined with L-tyrosine as a substrate under similar conditions. The kinetics course of oxidation of the substrate (monophenolase activity), in the presence of varied concentrations of cycloalliin, is shown in Figure 2B. The results confirmed that cycloalliin was a mixed-type inhibitor with a $K_{\mathrm{i}}$ value of $56.0 \mathrm{mM}$. It should be noted that arabinose also shows a mixed-type inhibitory effect on the oxidation of L-DOPA (diphenolase activity) with $K_{\mathrm{i}}$ value of $0.22 \mathrm{mM}(\mathrm{Hu}$ et al., 2012). Such a mixed mode of inhibition is frequently observed during kinetics studies on mushroom tyrosinase; nonspecific binding sites appear to exist on the protein structure of the enzyme and access to the active site, but this inhibition mechanism has not been elucidated to date (Hassani et al., 2016). Our results confirmed that cycloalliin had a subtle direct

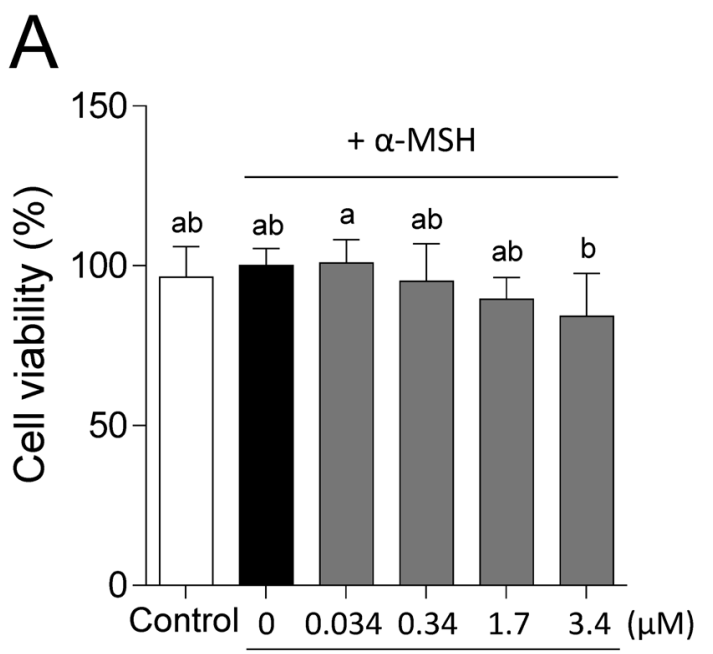

Final concentration of cycloalliin

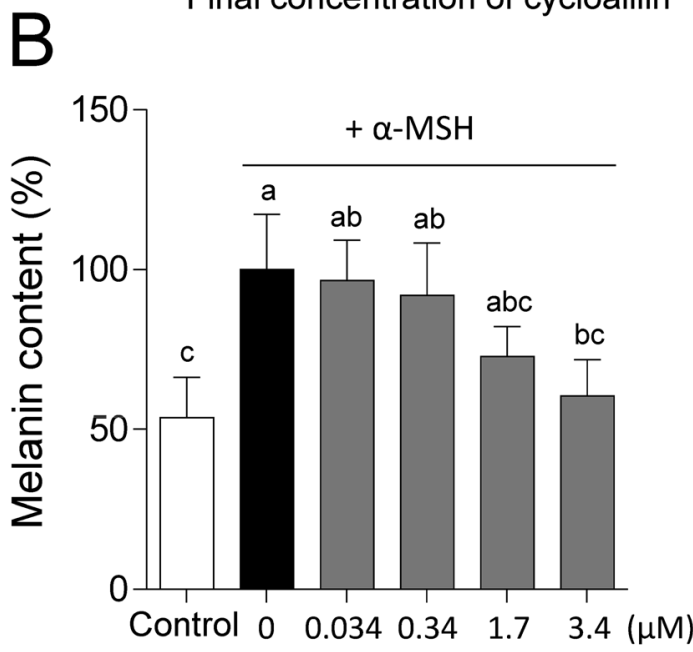

Final concentration of cycloalliin

Fig. 3. Effects of cycloalliin on $\alpha$-MSH-induced melanin synthesis in B16 mouse melanoma cells. (A) Cell viability; (B) Melanin content. The experiments were performed in triplicate. Data are expressed as percentage of the values in the cells treated with $0 \%$ cycloalliin. All values represent mean \pm SEM from three independent experiments. Different letters indicate values that are statistically significant $(p<0.05)$.

inhibition for mushroom tyrosinase.

Cycloalliin significantly reduced $\alpha$-MSH-induced melanin synthesis in B16 cells Cycloalliin showed no cytotoxic effects on B16 cells under the experimental conditions (Figure 3A). In the presence of $10 \mathrm{nM}$ of $\alpha-\mathrm{MSH}$, the melanin levels in B16 cells were significantly higher after $24 \mathrm{~h}$ than in its absence. When B16 cells were cultured in a medium supplemented with $10 \%(\mathrm{v} / \mathrm{v})$ cycloalliin (at a final concentration of $3.4 \mu \mathrm{M}$ ), $\alpha$-MSH-induced melanin production decreased significantly (Figure 3B).

Cycloalliin variably influenced mRNA levels of modulators and enzymes involved in cAMP-dependent melanogenesis The mRNA levels of tyrosinase, TRP1, TRP2, and MITF in homogenates of cells treated with the indicated amounts of 


\section{Tyrosinase}

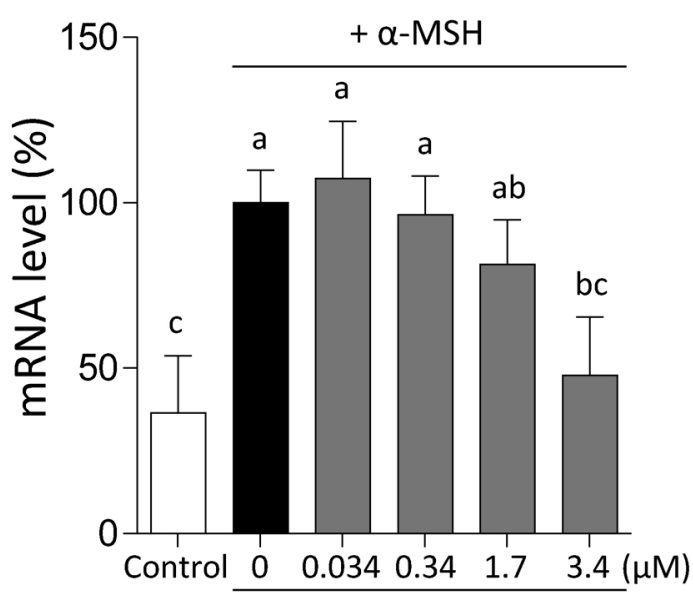

Final concentration of cycloalliin

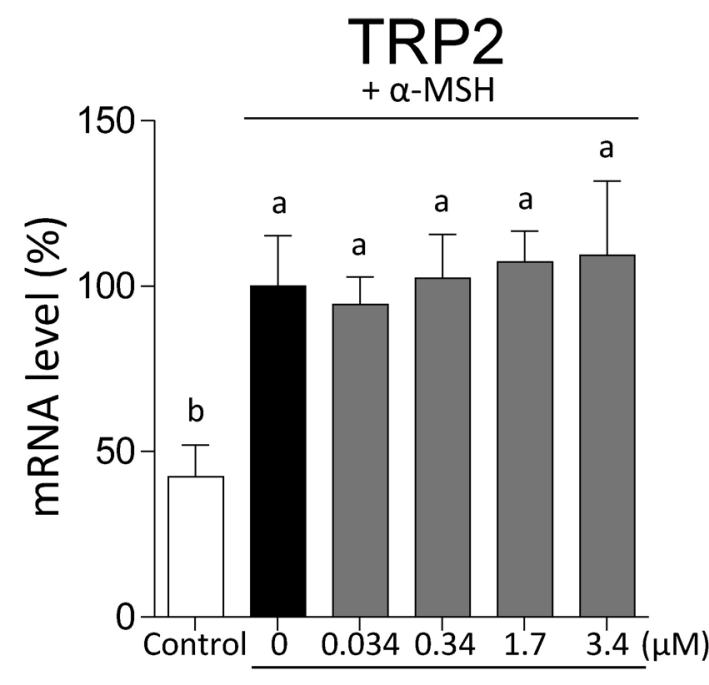

Final concentration of cycloalliin

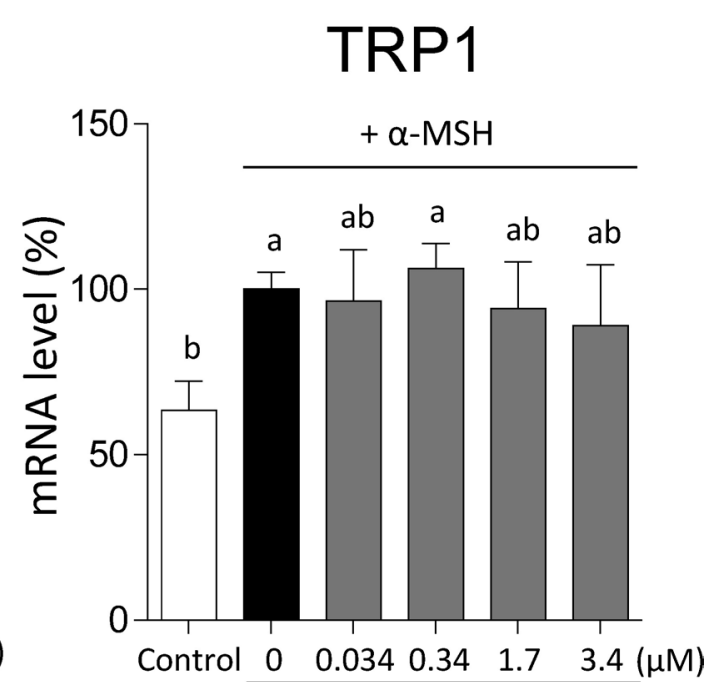

Final concentration of cycloalliin

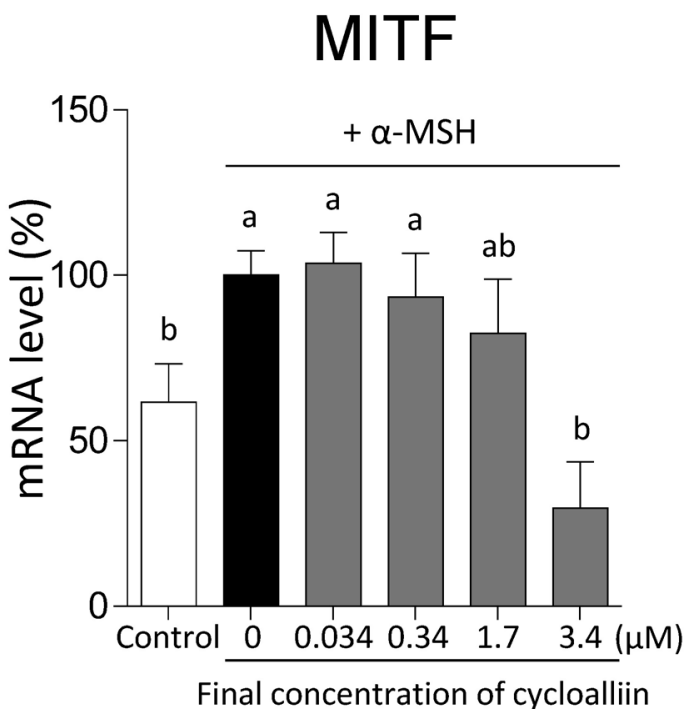

Fig. 4. The mRNA levels of tyrosinase, tyrosinase-related proteins, and microphthalmia-associated transcription factor in homogenates of the cells exposed to the indicated amounts of cycloalliin. Using qPCR, we determined the levels of mRNAs encoding tyrosinase, tyrosinase-related protein 1 (TRP1), tyrosinase-related protein 2 (TRP2), and microphthalmia-associated transcription factor (MITF). Data are expressed as percentages of the values in the cells treated with $0 \%$ cycloalliin. All values represent mean \pm SEM of three independent experiments; the different letters indicate values that are significantly different, $p<0.05$.

cycloalliin are presented in Figure 4. Cycloalliin $(3.4 \mu \mathrm{M})$ significantly decreased the expression of mRNAs encoding tyrosinase and MITF, but not those encoding TRP1 and TRP2 (Figure 4). It is unclear why the decreased mRNA levels of MITF did not affect both TRP1 and TRP2 mRNA levels. Lee et al. reported that glyceollin, a prenylated pterocarpan isolated from soybean, significantly decreases the levels of MITF, tyrosinase, and TRP1 in B16 melanoma cells but not those of TRP2 (Lee et al., 2010).

Cystamine, which is formed when cystine is thermally decarboxylated, has been shown to reduce MITF, tyrosinase, and TRP2 levels in SK-MEL-2 human melanoma cells, with the levels of TRP1 remaining unaffected (Kim et al., 2014).
Although $100 \mu \mathrm{M}$ of S-(-)-10,11-dihydroxyfarnesoic acid methyl ester (dhFAME) significantly inhibits the mRNA levels of tyrosinase, TRP1, TRP2, and MITF in murine melanocyte cells, only MITF mRNA expression was reduced when $50 \mu \mathrm{M}$ of dhFAME was used, and the levels of the rest of the proteins remained unchanged (Baek et al., 2014). Therefore, the inhibitors reducing MITF mRNA expression seem to trigger varied changes in the mRNA expression of other proteins.

The AC activity, which increased following the addition of $\alpha-\mathrm{MSH}$, decreased significantly enough to revert to the control levels in the cells treated with $10 \%(\mathrm{v} / \mathrm{v})$ cycloalliin (Figure 5A). Consequently, cAMP levels gradually decreased with a decrease in AC activity (Figure 5B). PKA activity reduced 

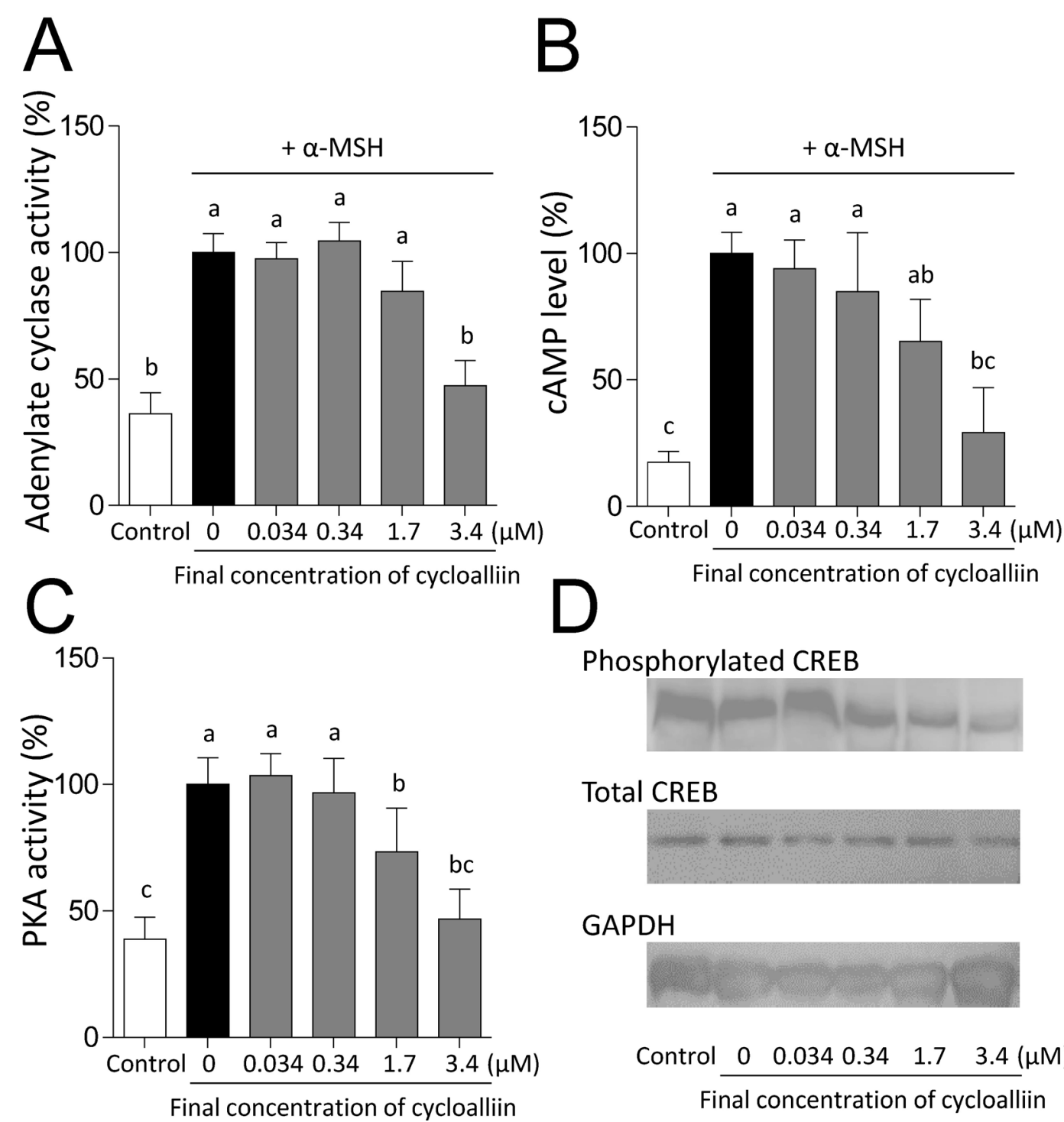

Final concentration of cycloalliin

Phosphorylated CREB

\section{Total CREB}

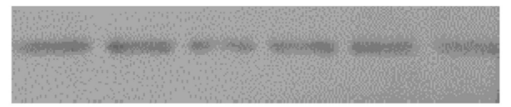

GAPDH

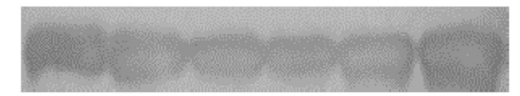

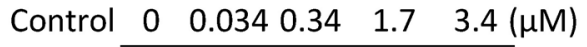

Final concentration of cycloalliin

Fig. 5. Effects of cycloalliin on cAMP-dependent signaling pathway in B16 mouse melanoma cells activated with $\alpha-M S H . ~(A)$ Adenylate cyclase activity, (B) cAMP levels, (C) PKA activity, and (D) phosphorylated CREB (western blot analysis using an anti-CREB phosphorylated antibody). Data are expressed as percentages of the values in the cells treated with $0 \%$ cycloalliin. All values represent mean \pm SEM of three independent experiments; the different letters indicate values that are statistically significant $(p<0.05)$.

significantly in a dose-dependent manner of cycloalliin concentration (Figurer 5C). In addition, the levels of phosphorylated CREB decreased significantly in the cells treated with $10 \%(\mathrm{v} / \mathrm{v})$ cycloalliin but cycloalliin did not affect the levels of total CREB (Figure 5D). These results indicate that cycloallin significantly decreased the mRNA levels of tyrosinase owing to the inhibition of $\mathrm{AC}$, following which the decreased activity of AC leads to the suppression of the cAMPsignaling pathway of melanogenesis. Coumarin (Kim et al., 2013) and 5,7-dihydroxyflavone (chrysin) (Kim et al., 2011), a potent $\mathrm{AC}$ inhibitor, block the binding of $\alpha-\mathrm{MSH}$ and forskolin as activators of $\mathrm{AC}$ to reduce melanin production through the cAMP- dependent signaling pathway in B16 cells.
It is reported that feeding cycloallin-containing diets to rats significantly reduce their serum triacylglycerol levels and attributed the phenomenon to cycloalliin-induced inhibition of the assembly of very low-density lipoproteins (VLDLs) (Yanagita et al., 2003). Recently, VLDL assembly was shown to be required for the processing of cAMP-responsive elementbinding protein $\mathrm{H}$ (Cheng et al., 2016). This suggests that cycloalliin could inhibit hepatic AC activity to decrease cAMP levels, leading to the inhibition of VLDL assembly. However, cycloalliin, as an AC inhibitor, cannot fully explain the increase of fibrinolytic activity in human blood (Agarwal et al., 1977) because the generation of cAMP inhibits platelet aggregation (Johnston-Cox et al., 2011). 
Our results showed that cycloalliin, which inhibited the catalytic activity of tyrosinase at high concentrations, mainly acts as a potent inhibitor of AC, which is involved in the cAMPsignaling pathway of melanogenesis, and then significantly decreases the mRNA expression of tyrosinase. Therefore, cycloalliin shows promise as a skin-lightening agent in the cosmetics industry.

Author contributions Tomohiro Bito, Kyohei Koseki, Tomohiro Moriguchi, and Yu Sasaki performed most experiments. Tsuyoshi Ichiyanagi contributed to chemical synthesis and identification of cycloalliin. Tomohiro Bito, Yukinori Yabuta, and Fumio Watanabe designed the experiments, interpreted the results, and wrote the manuscript. All authors commented on the manuscript and approved the final version.

Notes The authors declare no competing financial interests.

\section{References}

Agarwal, R.K., Dewar, H.A., Newell, D.L., and Das, B. (1977). Controlled trial of the effect of cycloalliin on the fibrinolytic activity of venous blood. Atherosclerosis, 27, 347-351.

Baek, S.H., Ahn, J.W., Nam, S.H., Yoon, C.S., Shin, J.C., and Lee, S.H. (2014). S-(-)-10,11-dihydroxyfarnesonic acid methyl ester inhibits melanin synthesis in murine melanocyte cells. Int. J. Mol. Sci., 15, 12750-12763.

Benítez, V., Mollá, E., Martín-Cabrejas, M.A., Aguilera, Y., LópezAndréu, F.J., Cools, K., Terry, L.A., and Esteban, R.M. (2011). Characterization of industrial onion wastes (Allium cepa L.): Dietary fibre and bioactive compounds. Plant Foods Hum. Nutr., 66, 48-57.

Cheng, D., Xu, X., Simon, T., Boudyguina, E., Deng, Z., VerHague, M., Lee, A.H., Shelness, G.S., Weinberg, R.B., and Parks, J.S. (2016). Very low density lipoprotein assembly is required for cAMP-responsive element-binding protein $\mathrm{H}$ processing and hepatic apolipoprotein A-IV expression. J. Biol. Chem., 291, 23793-23803.

Chang, T.S. (2012). Natural melanogenesis inhibitors acting through the down-regulation of tyrosinase activity. Materials, 5, 1661-1685.

Hassani, S., Haghbeen, K., and Fazli, M. (2016). Non-specific binding sites help to explain mixed inhibition in mushroom tyrosinase activities. Eur. J. Med. Chem., 122, 138-148.

Hu, W.J., Yan, L., Park, D., Jeong, H.O., Chung, H.Y., Yang, J.M., Ye, Z.M., and Qian, G.Y. (2012). Kinetic, structural and molecular docking studies on the inhibition of tyrosinase induced by arabinose. Int. J. Biol. Macromol., 50, 694-700.

Johnston-Cox, H.A., Yang, D., and Ravid, K. (2011). Physiological implications of adenosine receptor-mediated platelet aggregation. $J$. Cell Physiol., 226, 46-51.

Kameyama, K., Sakai, C., Kuge, S., Nishiyama, S., Tomita, Y., Ito, S., Wakamatsu, K., and Hearing, V.J. (1995). The expression of tyrosinase, tyrosinase-related proteins 1 and 2 (TRP1 and TRP2), the silver protein, and a melanoma cells of differing melanogenic activities. Pigment Cell Res., 8, 97-104.

Kim, D.C., Rho, S.H., Shin, J.C., Park, H.H., and Kim, D. (2011). Inhibition of melanogenesis by 5,7-dihydroxyflavone (chrysin) via blocking adenylyl cyclase activity. Biochem. Biophys. Res. Commun., 411, 121-125.

Kim, D.C., Rho, S.H., Kim, D., Kim, S.I., Jang, C.S., Ryu, J.K., Kim, B.W., Kweon, C.O., Kim, H.K., and Lee, S.J. (2013). Coumarin, a lead compound of warfarin, inhibits melanogenesis via blocking adenylyl cyclase. American Journal of Biomedical Research., 1, 43-47.

Kim, H.J., Lee, H.J., Park, M.K., Gang, K.J., Byun, H.J., Park, J.H., Kim, M.K., Kim, S.Y., and Lee, C.H. (2014). Involvement of transglutaminase-2 in $\alpha$-MSH-induced melanogenesis in SK-MEL-2 human melanoma cells. Biomol. Ther., 22, 207-212.

Kim, Y.J., No, J.K., Lee, J.H., and Chung, H.Y. (2005). 4,4'-Dihydroxybiphenyl as a new potent tyrosinase inhibitor. Biol. Pharm. Bull., 28, 323-327.

Lee, H.J., Suh, H.J., and Park, Y. (2013). Utilization of hydrolytic enzymes for the extraction of cycloalliin from garlic (Allium sativum L.). Proc. Biochem., 48, 1111-1117.

Lee, H.J., Suh, H.J., Han, S.H., Hong, J., and Choi, H.S. (2016) Optimization of extraction of cycloalliin form garlic (Allium sativum L.) by using principal components analysis. Prev. Nutr. Food Sci., 21, 138-146.

Lee, Y.S., Kim, H.K., Lee, K.J., Jeon, H.W., Cui, S., Lee, Y.M., Moon, B.J., Kim, Y.H., and Lee, Y.S. (2010). Inhibitory effect of glyceollin isolated from soybean against melanogenesis in B16 melanoma cells. BMB Rep., 43, 461-467.

Lineweaver, H. and Burk, D. (1934). The determination of enzyme dissociation constants. J. Am. Chem. Soc., 56, 658-666.

Loizzo, M.R., Tundis, R., and Menichini, F. (2012). Natural and synthetic tyrosinase inhibitors as antibrowning agents: An update. Com. Rev. Food Sci. Food Saf., 11, 378-398.

Sakai, K. and Yoneda, N. (1981). Convenient synthesis of 1,4-thiazane3-carboxylic acid derivatives. Chem. Pharm. Bull., 29, 1554-1560.

Ueda, Y., Tsubuku, T., and Miyajima, R. (1994). Composition of sulfur-containing components in onion and their flavor characters. Biosci. Biotech. Biochem., 58, 108-110.

Vazquez-Prieto, M.A. and Miatello, R.M. (2010). Organosulfur compounds and cardiovascular disease. Mol. Asp. Med., 31, $540-545$.

Xiao, H. and Parkin, K.L. (2002). Antioxidant functions of selected Allium thiosulfinates and S-alk (en) yl-L-cysteine sulfoxides. $J$. Agric. Food Chem., 50, 2488-2493.

Yanagita, T., Han, S.Y., Wang, Y.M., Tsuruta, Y., and Anno, T. (2003). Cycloalliin, a cyclic sulfur imino acid, reduces serum triacylglycerol in rats. Nutr., 19, 140-143. 